\title{
COVID-19 and Cleft and Craniofacial Surgery in Indian Scenario
}

\author{
Manikandhan Ramanathan ${ }^{1}$ - Sailesh Kumar Ravichandran \\ 1 (i) Anantanarayanan Parameswaran ${ }^{2}$
}

Received: 18 July 2020/ Accepted: 27 November 2020/Published online: 19 April 2021

(C) The Association of Oral and Maxillofacial Surgeons of India 2021

\begin{abstract}
The coronavirus disease (COVID-19) had created the new normal approach towards the management of all maxillofacial problems as it is highly contagious and causing a threat to the health care professionals. The surgical management of patients with cleft and craniofacial deformities has caused lots of anxiety among patients and doctors in the recent COVID era as some essential treatment will be required for cleft babies from day one. Safety and protection for cleft children and parents must be the priority while dealing with this non-emergency disease. This article will highlight the important steps of management of the cleft and craniofacial cases during this pandemic by adhering to the protocols. It also throws light towards the strategies in revoking the cleft surgical management at least till this infection subsides.
\end{abstract}

Keywords COVID-19 - Cleft surgery · Surgical protocols . New normal $\cdot$ Aseptic management . Cleft care

\section{Introduction}

The novel coronavirus SARS Cov-2 is a new strain of the virus with its continuously changing genetic virulence causing a lot of concern to health authorities. Lots of death among young adults have been reported attributing to the

Sailesh Kumar Ravichandran

drsailesh91@gmail.com

1 Meenakshi Cleft and Craniofacial Centre, Meenakshi General Hospital, Alapakkam Main Road, Maduravoyal, Chennai, Tamil Nadu 600 095, India

2 Meenakshi Ammal Dental College and Hospital, Chennai, India high virulence of mutating coronavirus. The route of spread is mainly through the airway by aerosol, and fomites from the inside infected persons [1]. The incubation period of the virus is ranging between 2 and 14 days with a mean of 5-6 days. The most common symptoms include fever, cough, shortness of breath, fatigue, loss of smell, and diarrhoea [2]. The pulmonary complication if severe can be fatal even after specific management. The risk of transmission is high as most of the patients are asymptomatic and cause the spread of the disease [2].

There is a high risk of COVID-19 virus transmission to the maxillofacial, ENT, and head and neck surgeons and dentists $[3,4]$. The majority of the countries had stopped doing non-essential surgeries due to the risk of contamination with the virus, but many European countries have started back cleft operations with adequate precautions and tests recently.

The social distancing and lockdowns have created a halt in the spread of major disasters in a country like India; with densely populated cities and lack of awareness among the people in urban and rural areas, it is likely to increase the incidence of cases in India. Though official figures reached one million affected when we write this, it will be a longlasting problem to the 1.3 billion Indian populations.

Though most of the cleft operations are done as a part of multispecialty set-ups in Indian hospitals, many of these already have enough COVID-19 infected patients either for monitoring or some on ventilation. It is possible in these situations that cross-contaminations during stay and surgery may make the patients and doctors prone to infect despite how well one maintains the protocols of prevention of contagion. 


\section{Challenges in Managing the Cleft and Craniofacial Patients During this Pandemic}

Prioritizing the surgical services at this moment of COVID-19 pandemic must be carefully balanced according to the patient's needs and availability of the resources. Postponement of cleft surgery should not affect the quality of life and speech outcomes especially in 1- or 2-year-olds with cleft palates. Authors tried to categorize in this write with the issues which can be pertinent to cleft centres in India or any developing country.

\section{Patient Mobilization from Camps and Other States}

Since most of the patients of the big centres in India rely on many camps conducted, in the present scenario it will be very difficult for village health care workers to travel and screen patients like before the COVID era. In cleft surgical set-ups where spontaneous reporting of patients outnumbers the patients reporting from camps, the situation may be a bit different. Despite the travel restriction within India, state to state, and inter-district, some patients tried to contact and reach cleft centres, though many preferred to wait and get it done on a later date.

Other issues patients facing are travel pass for a visit to hospital and return, fear of large incidences of corona in metro cities, and quarantine for a period of 7-14 days on long-distance travel between states.

\section{Digital Virtual Examination from Home}

In the present digital era, authors have used the WhatsApp web to effectively communicate with many parents of cleft children for counselling on treatment strategies and appointments for surgical works [5]. The WhatsApp video consultation also helped in scrutinizing the priorities of cleft surgery and other rehabilitation of patients at each age with the virtual examination of patients, understanding medical problems, and providing adequate time to make their children fit for surgeries.

The virtual meeting helped us also in getting the

(a) Demographic details of the patient, i.e., name, age, gender, location.

(b) To assess if a patient is having COVID-19 symptoms fever, cold, cough, and difficulty in breathing.

(c) Whether there is any family history of quarantine and local area.

(d) Any chance of infant infected from mother: detailed history discussed mothers' delivery details. (e) If they have registered with Arogya Setu app, then the records in it can be accessed from the patient's mobile.

\section{Laboratory Investigations}

Laboratory investigations routinely followed in our country is by taking nasal and oropharyngeal swabs, and RT-PCR is the gold standard for COVID-19 testing as recommended by the Indian Council of Medical Research (ICMR). The elevated CRP levels in the COVID 19 suspected patients also play a vital role in identifying the severity of the disease at the earliest [6].

Some studies also put forth the importance of the PA chest radiograph and CT chest to identify the hyalinised patches [7]; evaluation of the oxygen saturation levels also can act as an early indicator too [8].

Rapid point-of-care antigen detection test (PoC) can be used for large crowd where the results can be obtained within 15-30 min which helps us in screening before they have been brought in.

If a patient tests positive for COVID-19 [9], the choice of deferring the procedure should be taken by the surgeon after analysing the patient's needs until the patient is cured of COVID-19; this delay in surgery should not cause any irreparable damage to the patient.

After a one negative swab antibody test, the patient can be admitted or advised home quarantine for a minimum of 7 days to see whether the cleft child develops any signs of COVID-19 infection; during this period, the oxygen saturation has to be monitored everyday using a pulse oximeter, and if it is below $95 \%$ it has to be informed and a chest X-ray has to be done to assess the issue. Lung exercises are also advised with the use of respirometer in adult cleft patients. If fine after that period, repeat the chest X-ray and with the anaesthetic fitness, they can be taken up for the surgery with proper consent from the parents or patients of adult age.

\section{Updating the Consent Form}

The consent form in regional language should contain the following details,

- The risk of developing a COVID-19 infection during hospital stay during and after surgery.

- Limitations and prioritization regarding the essential surgical cases.

- Risks associated with not undertaking the surgery.

- The importance of adhering to social distancing guidelines. 
Clinicians should consider breaking language barriers and disabilities to reduce the risk of miscommunication [10].

\section{Prioritization of Procedures}

By bringing either 2 or 3 patients at one point of time with appointments, overcrowding of wards can be avoided to reduce transmission. After one batch of patients are discharged, the next batch can be brought in.

\section{Nasoalveolar Moulding and Feeding Plates}

If there is a necessity for nasoalveolar moulding in wide unilateral or bilateral cleft lip patients, this can be instituted as a priority if surgeons; this is a protocol which facilitates lip closure and nasal symmetry.

Being aware of the above-mentioned risk of virus transmission, the impression should be made in an aseptic method using an elastomeric impression material under the supervision of an anaesthetist done in the operating room with proper monitoring of the oxygen saturation during impression making. Immediately, the impression has to be disinfected by immersing in a glutaraldehyde or chlorine dioxide solution.

Use of $10 \%$ diluted betadine solution and subsequent wash with clean lukewarm water or normal saline for sterilizing the nasoalveolar moulding plate should be taught to the parents. It will be not possible to irrigate the mouth in babies as even minute amount of betadine aspiration may lead to atelectasis of lungs and acute respiratory failure.

\section{Severe Obstructive Sleep Apnoea Cases}

Craniofacial cases with the condition of Obstructive Sleep Apnoea (OSA) due to Pierre Robin sequence where retrognathia and glossoptosis are present, it is being recommended to perform mandibular distraction osteogenesis or tongue lip adhesion and they are considered to be a case of emergency depending on the severity in Apnoea-Hypopnea Index (AHI) [11]. Non-surgical methods like continuous positive pressure airway to be considered in mild cases of OSA, as the tracheostomy in such patients may pose a greater risk in contracting COVID-19.

\section{Speech Therapy}

Patients undergoing speech therapy with the speech pathologist can be done through video consultation, and recorded video messages can be sent to the parents as an activity to engage the cleft patient in proper speech training. Here also the WhatsApp or online video chat options come in handy between speech pathologist and patients/parents.

Middle ear infections in cleft palate patients can be coordinated with ear nose throat surgeons for grommets placement if there is severe otitis media. Even in these situations, initial antibiotic therapy and later corrections can be done on an appropriate date.

\section{Associated Treatment}

Apart from these priorities, no other treatment in cleft protocol will have emergencies for treatment including orthodontic therapy, bone grafting, osteotomes, and rhinoplasties. The cleft patients undergoing above management therapies are consulted through telephonic video, or surgery can be deferred untill the contagious infection comes down to a scenario where they can be safely treated as per the government's protocol.

\section{Maintaining Asepsis in the Operating Theatre- During Intubation}

During the surgical procedure under general anaesthesia, endotracheal intubation has to be done by adhering to the new protocols which were formulated and updated regularly during this new normal period; the anaesthetist and technical staffs during intubation are advised to wear the Personal Protection Equipment (PPE) and during intubation a transparent fibre barrier (aerosol box) to be placed between the anaesthetist and the patient (if available) to prevent aerosol spread from oropharynx and lungs. The use of video laryngoscope during intubation and the use of viral filters in the airway circuit avoids viral contact $[12,13]$.

In the case of anticipated difficult airway cases, the use of fibre-optic assisted awake intubation has to be performed with the strict protocol as the incidence of aerosol generation and circulation is very high.

The anaesthetist and the technical team is only allowed to be present in the operating room during intubation, and the operating team has to enter after 15-20 min after the intubation time; thus, the viral load in the operating room reduces by $99.9 \%$ as the operating room ventilation clears any potentially harmful aerosols in circulation [14].

\section{Revoking the Surgical Strategies for Cleft and Craniofacial Cases}

As per the directions of the union government, donning of PPE is mandatory during surgery. The cleft surgery should be considered as risky as any other airway surgery 
operation under the high-risk category. Doctors, nurses, and technical staff should strictly follow the procedures for donning and doffing the personal protective gear, and they should not be used beyond the contamination zone in the operating room [15].

Surgical procedures involving the use of electrocautery should be used minimally or avoided as it generates surgical smoke and advised the usage of high volume suction in the field of surgery [16]. It is quite difficult to completely avoid bipolar in cleft lip and palate surgeries, as it is primarily used for haemostasis to minimize blood loss and less postoperative issues in the paediatric group. Irrigation has to be done in drops over the tip of the cutting or bipolar tips to reduce inhalation aerosol.

If osteotomy is required, consider the use of osteotome and usage or bur at slow speed than a power saw or piezoelectric systems or high-speed drills. The author's opinion is that all these procedures can be till a convenient date to reduce aerosol in theatre, thereby avoiding inhalation by the surgical team, and also it will avoid fomites on the surface of the surgical room structures. The use of self-drilling screws is recommended in the case of orthognathic surgeries so that the aerosol generation can be reduced [3, 17]. Frequent forceful irrigation using saline should be avoided, and gentle wash is advised to prevent aerosol generation.

It is strongly recommended to use absorbable suture material for all surgical wounds to facilitate early discharge. Patients and the parents of cleft palate are taught to gently irrigate the surgical site intraorally using chlorhexidine to maintain the hygiene, thereby reducing the patient's risk of exposure to the contagious virus [18]. After the surgical procedure, the theatre is left vacant for around 15-20 min, as the ventilation removes the contaminants after which it can be cleaned and disinfected [19]. It is better to avoid surgeries back to back in the same operating room to reduce the risk of virus transmission.

At present, no guidelines were formulated to manage the cranial synostosis patients, acute intervention advised in case of increased intracranial pressure and papilledema $[11,20]$.

The use of non-steroidal anti-inflammatory drugs (NSAIDs) can increase the risk of adverse effects in COVID-19 patients; studies show mild to moderate effects with drug paracetamol in comparison to other NSAIDs; care should be taken to monitor for any adverse reactions [21].

\section{Perioperative Care in the Ward}

In the ward, strict aseptic protocols like handwashing and maintenance of social distancing should be followed among parents, cleft children, and paramedical staff.
Alternate beds should be kept empty and washrooms should be installed in multiple areas to prevent transmission of infection. The duration of hospital stays based on the patient's recovery should be minimized. The patient's attender should be restricted to one and visitors to the hospital avoided [22, 23].

When the cleft child gets discharged, parents are advised to continue the lip taping as taught. Regarding baby's feeding, the mother is advised to maintain personal hygiene every time during breastfeeding and also advised regarding the sterilization of the feeding bottles in a hot water bath or by the use of commercially available UV sterilizers.

Clinical photograph documentation of patients should be done by maintaining six feet distance from the photograph spot, and the patient is made to gargle using $0.2 \%$ betadine mouthwash for $30 \mathrm{~s}$, and intraoral photographs to be taken thereafter $[1,24]$. The intraoral mouth mirrors and cheek retractors are properly sterilized before to the next photograph taken to avoid cross-contamination.

All health care professionals and patient attendees should be clinically screened for fever and oxygen saturation every day [9]. The disinfectants like $1 \%$ sodium hypochlorite or alcohol-based disinfectants should be frequently used in the wards at least twice in a day.

\section{Prophylaxis for Doctors and Frontline Health Workers}

As per the advice of the Indian Council of Medical Research (ICMR), the use of hydroxychloroquine (HCQ) should be as prophylaxis for healthcare professionals, as various observational studies showed that those taking HCQ prophylaxis had a lower incidence of SARS-CoV-2 infection than those not taking it. ICMR has advised a dose of $400 \mathrm{mg}$ twice on day 1 and $400 \mathrm{mg}$ once weekly for the next 7 weeks, to be taken with meals [25].

The medication is contraindicated in people with documented cases of retinopathy, HCQ hypersensitivity or 4-aminoquinoline compounds, G6PD deficiency, pre-existing cardiomyopathy, and heart rhythm disorders [12].

\section{Summary of Key Strategies During COVID-19 Time Cleft Surgeries}

1. Telephonic/video consultation and counselling before the clinical examination.

2. Preoperative consultation by the surgeon and anaesthetist by donning PPE.

3. As per the Indian government guidelines on screening, the patient should have at least one negative 
swab and an antibody test and should obtain special COVID-19 consent as per the institution guidelines.

4. To follow intubation guidelines and to avoid generating aerosols during such procedures by the usage of blocks and video laryngoscopes.

5. The operating room should not be opened frequently during the procedure, and all required materials to be arranged before the procedure.

6. All members of the surgical team should wear an N95 mask or a similar protection respirator preferably half or full masks with filters.

7. To avoid irrigation frequently and use of high-speed bur not advocated and high-pressure suction devise to be used to avoid aerosol during the procedure.

8. The use of absorbable sutures is recommended so as to prevent the patients' visit later.

9. The cleaning of the theatre room should be done after 20-30 min, as the room ventilation clears all the potentially harmful aerosol.

10. Minimizing the postoperative stays in the hospital.

\section{Conclusion}

There is always an ambiguity on how long this corona scenario will continue in India. Therefore, the final treatment decision depends on the institution and the surgeon towards the betterment of the patient, as most of the cleft and craniofacial surgeries are time-sensitive. It is necessary to provide some emergency surgery for these patients with at most precautions to the patient and surgical team.

Availability of Data and Materials As quoted in the reference section.

\section{Compliance with Ethical Standards}

Conflict of interest All authors declare that they have no conflict of interest.

\section{References}

1. Peng X, Xu X, Li Y, Cheng L, Zhou X, Ren B (2020) Transmission routes of 2019-nCoV and controls in dental practice. Int $\mathrm{J}$ Oral Sci 12:9. https://doi.org/10.1038/s41368-020-0075-9

2. Singhal T (2020) A review of coronavirus disease-2019 (COVID19). Indian J Pediatr 87:281-286. https://doi.org/10.1007/s12098020-03263-6

3. Givi B, Schiff BA, Chinn SB, Clayburgh D, Iyer NG, Jalisi S, Moore MG, Nathan CA, Orloff LA, O'Neill JP, Parker N, Zender C, Morris LGT, Davies L (2020) Safety recommendations for evaluation and surgery of the head and neck during the COVID19 pandemic. JAMA Otolaryngol Head Neck Surg. https://doi. org/10.1001/jamaoto.2020.0780
4. Zimmermann M, Nkenke E (2020) Approaches to the management of patients in oral and maxillofacial surgery during COVID19 pandemic. J Craniomaxillofac Surg. https://doi.org/10.1016/j. jcms.2020.03.011

5. Car J, Koh GC-H, Foong PS, Wang CJ (2020) Video consultations in primary and specialist care during the Covid-19 pandemic and beyond. BMJ 371:3945. https://doi.org/10.1136/bmj. m3945

6. Ling W (2020) C-reactive protein levels in the early stage of COVID-19. Med Mal Infect. https://doi.org/10.1016/j.medmal. 2020.03.007

7. Fang Y, Zhang H, Xie J et al (2020) Sensitivity of Chest CT for COVID-19: comparison to RT-PCR. Radiology 296(2):E115E117. https://doi.org/10.1148/radiol.2020200432

8. Kang S, Peng W, Zhu Y et al (2020) Recent progress in understanding 2019 novel coronavirus (SARS-CoV-2) associated with human respiratory disease: detection, mechanisms and treatment. Int J Antimicrob Agents 55(5):105950. https://doi.org/10.1016/j. ijantimicag.2020.105950

9. Guidelines for safe ENT practice in COVID-19. Ministry of health and family welfare, India. https://www.mohfw.gov.in/pdf/ ENTCOVID0306.pdf. Accessed date 19 June 2020

10. Al-Jabir A, Kerwan A, Nicola M et al (2020) Impact of the Coronavirus (COVID-19) pandemic on surgical practice-part 2 (surgical prioritisation). Int J Surg 79:233-248. https://doi.org/10. 1016/j.ijsu.2020.05.002

11. Schoenbrunner A, Sarac B, Gosman A, Janis JE (2020) Considerations for pediatric craniofacial surgeons during the COVID-19 outbreak. J Craniofacial Surg. https://doi.org/10.1097/SCS. 0000000000006565

12. Begley JL, Lavery KE, Nickson CP, Brewster DJ (2020) The aerosol box for intubation in coronavirus disease 2019 patients: an in situ simulation crossover study. Anaesthesia 75:1014-1021. https://doi.org/10.1111/anae.15115

13. Peng PWH, Ho P-L, Hota SS (2020) Outbreak of a new coronavirus: what anaesthetists should know. $\mathrm{Br} \mathrm{J}$ Anaesth 124(5):497-501. https://doi.org/10.1016/j.bja.2020.02.008

14. AO CMF International Task Force (2020) AO CMF International Task Force recommendations on best practices for maxillofacial procedures during COVID-19 pandemic. https://aocmf3.aofoun dation.org/-/media/project/aocmf/aocmf/files/covid-19/ao_cmf_ covid-19_task_force_guidelines. Accessed date 4 July 2020

15. Liu Z, Zhang Y, Wang X et al (2020) Recommendations for surgery during the novel coronavirus (COVID-19) epidemic. Indian J Surg 82:124-128. https://doi.org/10.1007/s12262-02002173-3

16. Wang TT, Moon HS, Le A, Panchal N (2020) Proceedings of the OMS COVID-19 Response Conference. J Oral Maxillofac Surg 78(8):1268-1274. https://doi.org/10.1016/j.joms.2020.05.003

17. Interim infection prevention and control recommendations for patients with suspected or confirmed coronavirus disease 2019 (COVID-19) in healthcare settings. cdc.gov. https://www.cdc. gov/coronavirus/2019-ncov/hcp/infection-controlrecommenda tions.html, Accessed date 7 April 2020

18. Keny S, Bagaria V, Chaudhary K, Dhawale A (2020) Emergency and urgent orthopaedic surgeries in non-Covid patients during the COVID 19 pandemic: perspective from India. J Orthop 20:275-279. https://doi.org/10.1016/j.jor.2020.05.012

19. Van Doremalen N, Bushmaker T, Morris DH et al (2020) Aerosol and surface stability of SARS-CoV-2 as compared with SARSCoV-1. N Engl J Med. https://doi.org/10.1056/NEJMc2004973

20. ACPA Statement on the Reactivation of Cleft and Craniofacial Care During the COVID-19 Pandemic. https://acpa-cpf.org/wpcontent/uploads/2020/05/ACPA-Position-Statement-on-Reactiva tion.pdf. Accessed date 19 May 2020 
21. Paul Little (2020) Non-steroidal anti-inflammatory drugs and Covid-19. BMJ 368:m1185. https://doi.org/10.1136/bmj.m1185

22. Preparing for COVID-19 in nursing homes. https://www.cdc.gov/ coronavirus/2019-ncov/hcp/long-term-care.html. Accessed date 4 July 2020

23. Maffia F, Fontanari M, Vellone V, Cascone P, Mercuri LG (2020) Impact of COVID-19 on maxillofacial surgery practice: a worldwide survey. Int $\mathbf{J}$ Oral Maxillofac Surg 49:827-835. https://doi.org/10.1016/j.ijom.2020.04.015

24. Khan FR (2020) A clinical trial of gargling agents in reducing intraoral viral load among COVID-19 patients (GARGLES).
https://clinicaltrials.gov/ct2/show/NCT04341688. Accessed date 30 June 2020

25. Revised advisory on the use of Hydroxychloroquine (HCQ) as prophylaxis for SARS-CoV-2 infection. https://www.icmr.gov.in/ pdf/covid/techdoc/V5_Revised_advisory_on_the_use_of_HCQ_ SARS_CoV2_infection.pdf. Accessed 20 June 2020

Publisher's Note Springer Nature remains neutral with regard to jurisdictional claims in published maps and institutional affiliations. 